Article

\title{
Changes of Photosynthetic Behaviors and Photoprotection during Cell Transformation and Astaxanthin Accumulation in Haematococcus pluvialis Grown Outdoors in Tubular Photobioreactors
}

\author{
Litao Zhang ${ }^{1,2,3}$, Fang Su 1,4, Chunhui Zhang ${ }^{1,4}$, Fengying Gong ${ }^{1,4}$ and Jianguo Liu 1,2,3,* \\ 1 Key Laboratory of Experimental Marine Biology, Institute of Oceanology, Chinese Academy of Sciences, \\ Qingdao 266071, China; zhanglitao666@163.com (L.Z.); sufang116@126.com (F.S.); \\ zhangchunhui13@mails.ucas.ac.cn (C.Z.); 18766216016@163.com (F.G.) \\ 2 National-Local Joint Engineering Research Center for Haematococcus pluvialis and Astaxanthin, \\ Yunnan Alphy Biotech Co., Ltd., Chuxiong 675012, China \\ 3 Laboratory for Marine Biology and Biotechnology, Qingdao National Laboratory for Marine Science \\ and Technology, Qingdao 266071, China \\ 4 Institute of Oceanology, University of Chinese Academy of Sciences, Beijing 100049, China \\ * Correspondence: jgliu@qdio.ac.cn; Tel.: +86-532-8289-8709; Fax: +86-532-8289-8612
}

Academic Editor: Yongsheng Chen

Received: 23 October 2016; Accepted: 20 December 2016; Published: 26 December 2016

\begin{abstract}
The cell transformation from green motile cells to non-motile cells and astaxanthin accumulation can be induced in the green alga Haematococcus pluvialis cultured outdoors. In the initial $3 \mathrm{~d}$ of incubation (cell transformation phase), light absorption and photosynthetic electron transport became more efficient. After five days of incubation (astaxanthin accumulation phase), the light absorption per active reaction center (ABS/RC) increased, but the efficiency of electron transport $\left(\psi_{0}\right)$ and the quantum yield of electron transport $\left(\varphi_{E o}\right)$ decreased with increased time, indicating that the capacity of photosynthetic energy utilization decreased significantly during astaxanthin accumulation, leading to an imbalance between photosynthetic light absorption and energy utilization. It would inevitably aggravate photoinhibition under high light, e.g., at midday. However, the level of photoinhibition in $\mathrm{H}$. pluvialis decreased as the incubation time increased, which is reflected by the fact that $F_{v} / F_{m}$ determined at midday decreased significantly in the initial $3 \mathrm{~d}$ of incubation, but was affected very little after seven days of incubation, compared with that determined at predawn. This might be because the non-photochemical quenching, plastid terminal oxidase, photosystem I cyclic electron transport, defensive enzymes and the accumulated astaxanthin can protect cells against photoinhibition.
\end{abstract}

Keywords: astaxanthin; chlorophyll a fluorescence transient; Haematococcus pluvialis; photoinhibtion; photosynthetic behavior

\section{Introduction}

Haematococcus pluvialis, a unicellular green alga, is widely known for its ability to accumulate large amounts of astaxanthin (3,3'-dihydroxy- $\beta, \beta$-carotene- $4,4^{\prime}$-dione) [1-3]. The astaxanthin is used not only as a pigmentation source in aquaculture and poultry industries [4,5], but also has potential clinical applications due to its antioxidant activity, which is higher than that of $\beta$-carotene and $\alpha$-tocopherol [6,7]. A two-step culture protocol is developed to cultivate $H$. pluvialis for astaxanthin production [8]. In the first step, favorable growth conditions are provided to accelerate H. pluvialis 
cell growth and to obtain a high biomass (cell growth phase); in the second step, stress conditions are provided to induce cell transformation from green motile cells to non-motile cells (cell transformation phase), and to induce the accumulation of astaxanthin (astaxanthin accumulation phase).

The cell transformation and astaxanthin accumulation are strongly light-induced processes for H. pluvialis, and can be regulated by photosynthetic redox status [9-11]. Therefore, understanding the changes of photosynthetic behaviors during the cell transformation and astaxanthin accumulation would provide some new insights into mechanisms of astaxanthin accumulation in H. pluvialis. However, most of the work published so far on $H$. pluvialis has been focused on the optimization of cell growth and the induction of astaxanthin accumulation of this alga [3,12]. Far less attention has been paid to the changes of photosynthetic behaviors, especially the energy fluxes of absorption, trapping and electron transport, in H. pluvialis during cell transformation and astaxanthin accumulation. Until recently, many works about the response of photosynthesis during cell transformation and astaxanthin accumulation in $H$. pluvialis grown indoors have been reported. Scibilia et al. studied the photosynthetic response to nitrogen starvation and high light in H. pluvialis [13]. Chekanov et al. investigated the modulation of photosynthetic activity in H. pluvialis during the incubation [14]. However, there is no work concerning outdoor photosynthetic response of this species reported.

The chlorophyll $a$ fluorescence transient (labeled also as OJIP transient, because the fluorescence transient shows a polyphasic rise including phase $\mathrm{O}, \mathrm{J}$, I and $\mathrm{P}$ ) combined with a JIP-test is one of the most popular tools in photosynthetic research [15-18]. It is possible to calculate several phenomenological and biophysical expressions of photosynthetic behaviors [19-21]. In the present study, we used the OJIP transient combined with the JIP-test to detect changes in photosynthetic behaviors, including the energy fluxes of absorption, trapping, and electron transport during the cell transformation and astaxanthin accumulation in H. pluvialis grown outdoors in tubular photobioreactors. Meanwhile, changes of photoprotective mechanisms during the cell transformation and astaxanthin accumulation were also analyzed. Determining the changes of photosynthetic behaviors and photoprotection during cell transformation and astaxanthin accumulation in H. pluvialis grown outdoors would help in optimizing astaxanthin production.

\section{Results}

In tubular photobioreactors outdoors (Figure 1A,B), H. pluvialis cells underwent a morphological change over time (Figure 1B,C). The green motile cells lost their flagella and motility and then transformed into larger, non-motile cells during the first three days of incubation (cell transformation phase). Astaxanthin started to accumulate after three days of incubation (astaxanthin accumulation phase). The accumulation of astaxanthin in H. pluvialis was significant $(p<0.05)$ after five days of incubation (Figure 1C). The astaxanthin content increased from $0.47 \mu \mathrm{g} \cdot \mathrm{mL}^{-1}\left(0.02 \mu \mathrm{g} \cdot 10^{4} \mathrm{cells}^{-1}\right)$ to $12.89 \mu \mathrm{g} \cdot \mathrm{mL}^{-1}\left(0.56 \mu \mathrm{g} \cdot 10^{4} \mathrm{cells}^{-1}\right)$ during a nine-day incubation period (Figure 1D,E). The astaxanthin content on cellular dry weight increased from $0.11 \%$ to $0.97 \%$ (data not shown). Chlorophyll content increased gradually with time during the initial seven days of incubation, but decreased slightly after seven days of incubation in H. pluvialis (Figure 1D,E).

The respiration rate in $H$. pluvialis grown outdoors in tubular photobioreactors did not change during the incubation. The total $\mathrm{O}_{2}$ evolution rate, which is an indicator of photosynthetic capacity in plants, enhanced in the initial three days of incubation and decreased gradually over time after five days of incubation (Figure 2). 

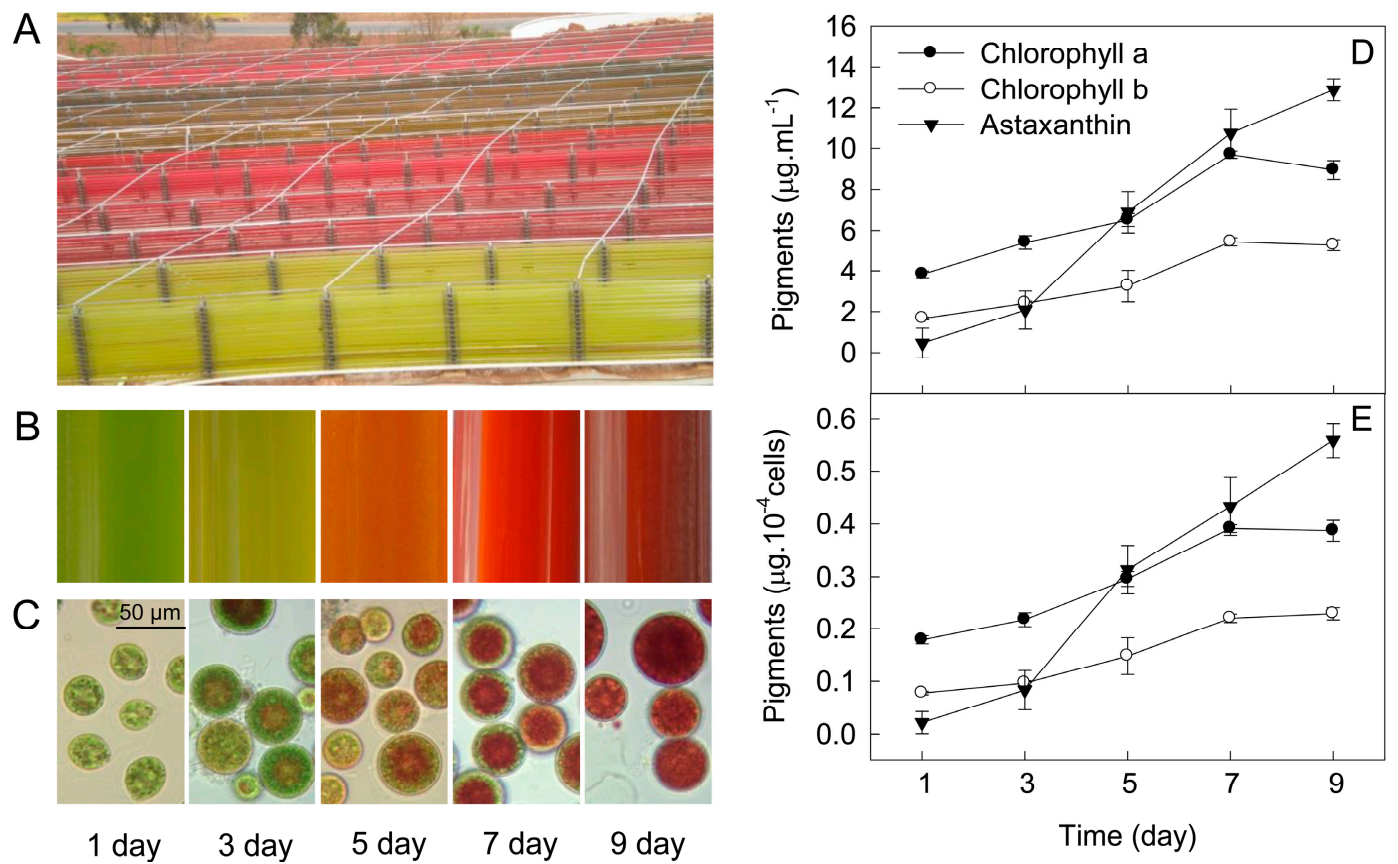

Figure 1. The processes of cell transformation and astaxanthin accumulation in H. pluvialis grown outdoors in tubular photobioreactors $(\mathbf{A}, \mathbf{B})$; The cell morphology during the cell transformation and astaxanthin accumulation in H. pluvialis in the tubular photobioreactors (C); The changes of chlorophyll and astaxanthin contents during cell transformation and astaxanthin accumulation in $H$. pluvialis cultured in tubular photobioreactors $(D, E)$. Mean \pm SE of five replicates are presented.

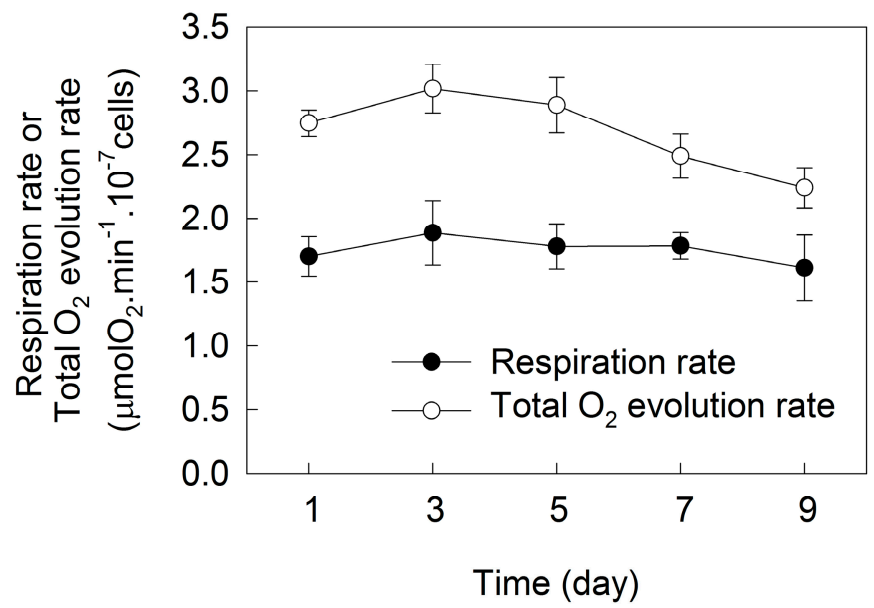

Figure 2. The respiration rate and total $\mathrm{O}_{2}$ evolution rate during cell transformation and astaxanthin accumulation in $H$. pluvialis cultured in tubular photobioreactors. Mean \pm SE of five replicates are presented.

The changes of chlorophyll $a$ fluorescence (OJIP) transients during the incubation in H. pluvialis cells are depicted (Figure 3A). The H. pluvialis cells showed a typical OJIP chlorophyll fluorescence transient (the $\mathrm{O}, \mathrm{J}, \mathrm{I}$, and $\mathrm{P}$ steps are marked in the plot) at one day. The variation in the shape of OJIP transients was obvious with increased time during the incubation (Figure 3A), suggesting that photosystem (PS) II behaviors were changed during cell transformation and astaxanthin accumulation. 

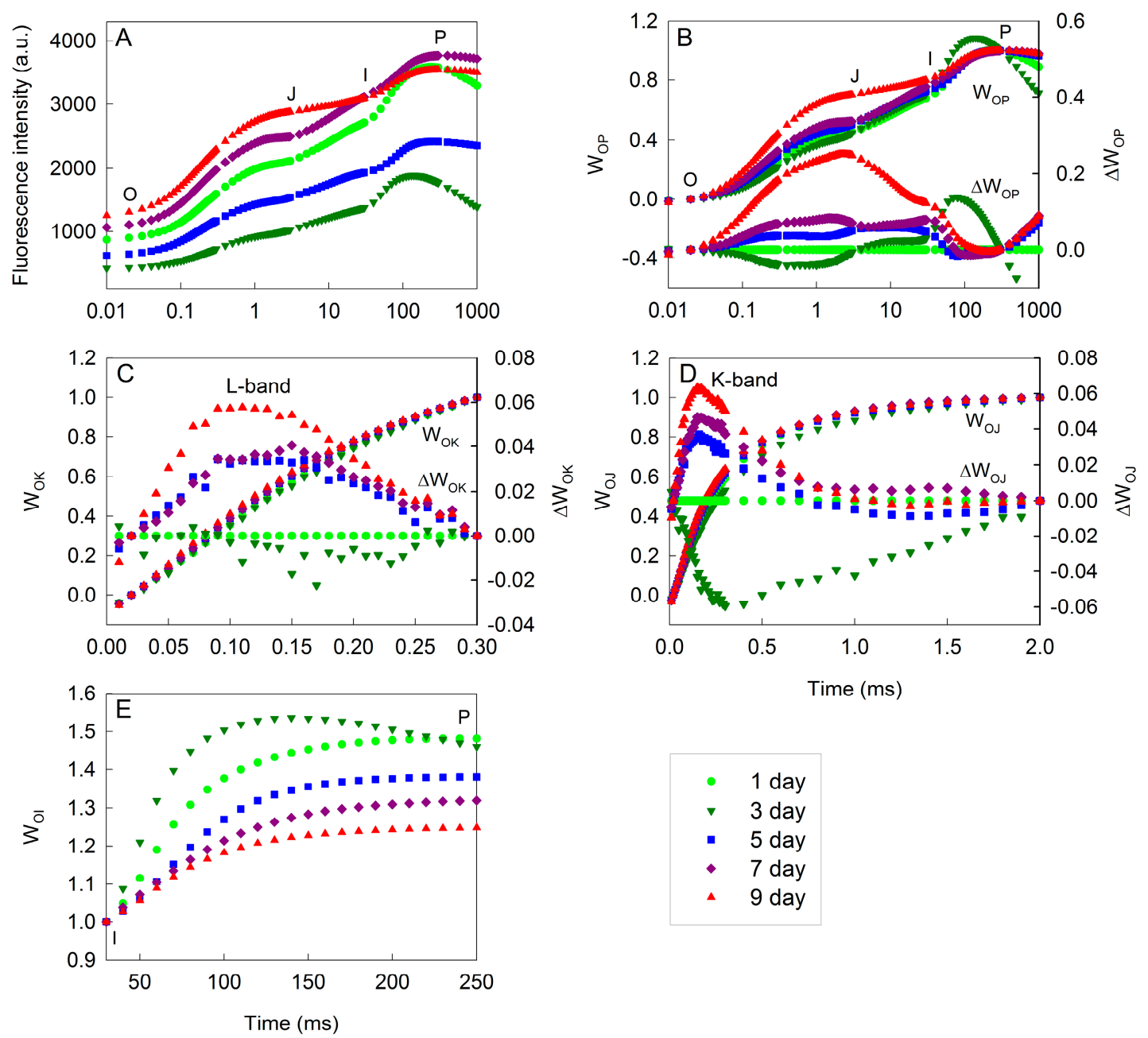

$$
\begin{aligned}
& \text { - } 1 \text { day } \\
& \text { - } 3 \text { day } \\
& \text { - } 5 \text { day } \\
& \text { - } 7 \text { day } \\
& \text { - } 9 \text { day }
\end{aligned}
$$

Figure 3. The chlorophyll $a$ fluorescence (OJIP) transients during cell transformation and astaxanthin accumulation in $H$. pluvialis cultivated in tubular photobioreactors (A); the normalization of fluorescence transients between the O-P (B), O-K (C), O-J (D) and O-I (E) phases. The O, J, I, and P steps are marked in the plot. The corresponding difference kinetics between cells incubated for three, five, seven or nine days and cells incubated for one day $\left(\Delta W=W-W_{\text {(cells incubated for } 1 \text { day })}\right)$ are also depicted. Each transient represents the average of eight samples. The difference kinetics $\Delta W_{O J}$ and $\Delta W_{O K}$ reveal the $K$-band (at about $300 \mu \mathrm{s}$ ) and $L$-band (at about $150 \mu \mathrm{s}$ ), respectively.

To clarify the detailed changes of PSII behaviors during the incubation in $H$. pluvialis, the changes in the phases of O-P, O-K, O-J and O-I in the relative variable fluorescence kinetics were analyzed by normalizing the OJIP transients (Figure 3B-E). It shows that fluorescence transients normalized between $\mathrm{O}$ and $\mathrm{P}$ were changed greatly with increased time during the incubation. The most distinct peak in the relative variable fluorescence kinetics was at the J-step (at about $2 \mathrm{~ms}$ ), which increased with increased time after five days of incubation (Figure 3B), indicating that the ratio of $\mathrm{Q}_{\mathrm{A}}{ }^{-} / \mathrm{Q}_{\mathrm{A}}$ had increased $[18,19]$. In contrast, the J-step did not change in the first three days of incubation.

The $L$-bands did not change in the initial three days of incubation, and increased significantly $(p<0.05)$ over time after five days of incubation (Figure 3C). The higher L-band (Figure 3C) indicates a lower energetic connectivity of the PSII units, which results in a poorer utilization of the excitation energy and a lower stability of the system [17,18]. The K-bands (Figure 3D) decreased during the initial three days of incubation, and increased significantly $(p<0.05)$ after five days of incubation. The enhanced K-band indicates increased damaged to the donor side of PSII [15,17]. 
The maximal amplitude of fluorescence in the I-P phase of the OJIP transient reflects the pool size of end electron acceptors $[17,18]$. The maximal amplitudes of fluorescence in the I-P phase increased during the initial three days of incubation, and decreased significantly $(p<0.05)$ in H. pluvialis over time after five days of incubation (Figure 3E).

The light absorption per active reaction center (ABS/RC, the average antenna size) and the trapping of excitation energy per active reaction center (TRo/RC) decreased slightly, the efficiency of electron transport $\left(\psi_{0}\right)$ and the quantum yield of electron transport $\left(\varphi_{E o}\right)$ increased, and the electron transport per active reaction center $(\mathrm{ETo} / \mathrm{RC})$ did not change over time during the initial three days of incubation (Figure 4). This suggests that the capacities of light absorption and photosynthetic electron transport were highly efficient $[17,22]$ in $H$. pluvialis during the first three days of incubation. However, the ABS/RC increased, whereas the ETo/RC, $\psi_{o}, \varphi_{E o}$ decreased, over time after five days of incubation. The ABS/RC increased about $26.7 \%$, whereas the ETo/RC, $\psi_{o}$ and $\varphi_{E o}$ decreased about $41.3 \%, 45.2 \%$ and $53.5 \%$, respectively, during a nine-day incubation period.

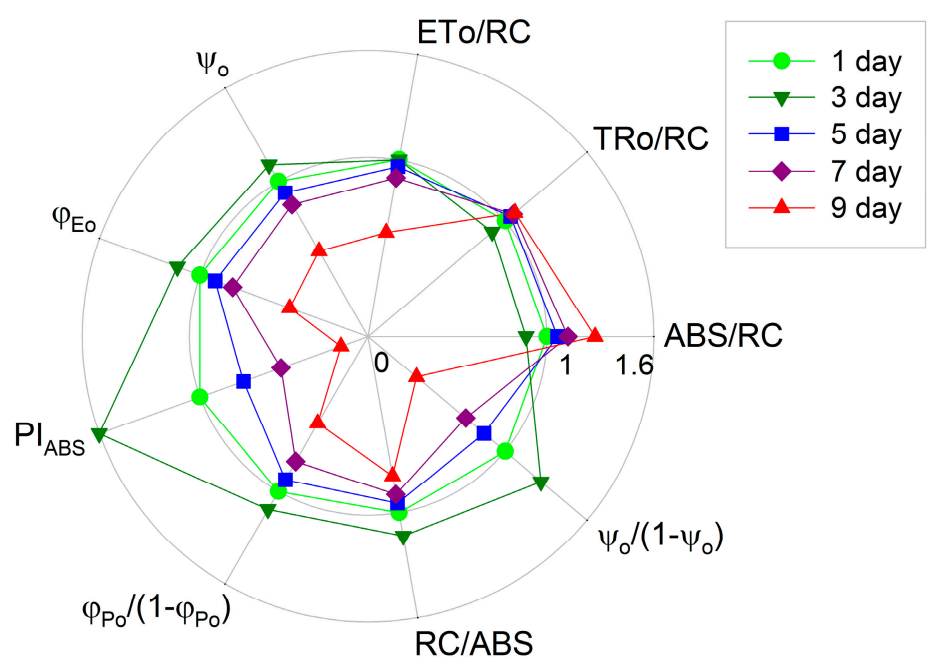

Figure 4. The light absorption per active reaction center (ABS/RC, the average antenna size), the trapping of excitation energy per active reaction center (TRo/RC), the electron transport per active reaction center $(\mathrm{ETo} / \mathrm{RC})$, the efficiency of electron transport $\left(\psi_{0}\right)$, the quantum yield of electron transport $\left(\varphi_{E o}\right)$ and the performance index $\left(\mathrm{PI}_{\mathrm{ABS}}\right)$ as well as its individual partial components RC/ABS, $\varphi_{P_{0}} /\left(1-\varphi_{P_{0}}\right)$, and $\psi_{o} /\left(1-\psi_{0}\right)$, during cell transformation and astaxanthin accumulation in H. pluvialis grown in tubular photobioreactors. Each point represents the average of eight samples. All parameters in H. pluvialis cells incubated for one day were taken as one, whereas those in cells incubated for three, five, seven or nine days were expressed as a percentage of these parameters in cells incubated for one day. The original values of ABS/RC, TRo/RC, ETo/RC, $\psi_{o}, \varphi_{E o}, \mathrm{PI}_{\mathrm{ABS}}, \mathrm{RC} / \mathrm{ABS}, \varphi_{P_{0}} /\left(1-\varphi_{P_{o}}\right)$ and $\psi_{0} /\left(1-\psi_{0}\right)$ in the cells incubated for one day were $3.19 \pm 0.02,2.45 \pm 0.02,1.35 \pm 0.04,0.55 \pm 0.02$, $0.42 \pm 0.01,1.27 \pm 0.03,0.31 \pm 0.00,3.31 \pm 0.17$ and $1.23 \pm 0.10$, respectively.

The performance index $\mathrm{PI}_{\mathrm{ABS}}$ is closely related to energy conservation capability and the activity of the photosynthetic apparatus $[17,18,21]$. Because it incorporates several parameters that are evaluated from the OJIP transient, it is the most sensitive indicator reflecting the photosynthetic performance. The $\mathrm{PI}_{\mathrm{ABS}}$ in $H$. pluvialis increased significantly $(p<0.05)$ in the initial three days of incubation, and declined gradually over time after five days of incubation (Figure 4). The component parameters of $\mathrm{PI}_{\mathrm{ABS}}$, the efficiency of absorption of light (RC/ABS), the performance due to the quantum efficiency of primary photochemistry $\left(\varphi_{P_{0}} /\left(1-\varphi_{P_{0}}\right)\right)$ and the performance due to the quantum efficiency of the conversion of excitation energy to electron transport $\left(\psi_{0} /\left(1-\psi_{0}\right)\right)$, increased significantly during the initial three days of incubation, and declined gradually over time after five days of incubation (Figure 4). The $\mathrm{PI}_{\mathrm{ABS}}$ decreased by $84.0 \%$ in H. pluvialis during a nine-day incubation period. The individual effect 
on the component parameters of $\mathrm{PI}_{\mathrm{ABS}}$ during a nine-day incubation period was as follows: $\mathrm{RC} / \mathrm{ABS}$ decreased by $21.0 \%, \varphi_{P_{0}} /\left(1-\varphi_{P_{0}}\right)$ decreased by $43.7 \%$, and $\psi_{0} /\left(1-\psi_{0}\right)$ decreased by $64.7 \%$ (Figure 4 ). It seems that the contribution of the conversion of excitation energy to electron transport was the most sensitive partial component with increased time during the incubation of $H$. pluvialis.

To investigate the change of PSI activity during cell transformation and astaxanthin accumulation in H. pluvialis grown outdoors, the level of PSI core protein (PsaA) was analyzed. The level of PsaA increased in the initial three days of incubation, and declined gradually over time after five days of incubation (Figure 5).

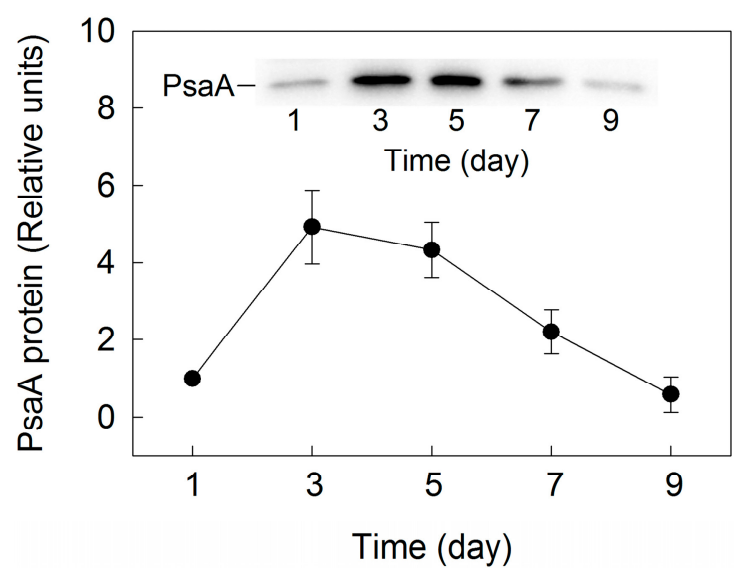

Figure 5. Densitometry of photosystem I core protein protein (PsaA) during cell transformation and astaxanthin accumulation in $\mathrm{H}$. pluvialis cultured in tubular photobioreactors. Inset shows the levels of PsaA protein. Representative results of three independent replicates are shown.

Non-photochemical quenching (NPQ) in H. pluvialis decreased gradually during the incubation (Figure 6). The fast component of NPQ $(q \mathrm{E})$ did not change in the initial three days of incubation, and decreased slightly over time after five days of incubation. The slow component of NPQ $(q \mathrm{I})$ decreased sharply in the initial three days of incubation, and did not change over time after five days of incubation.

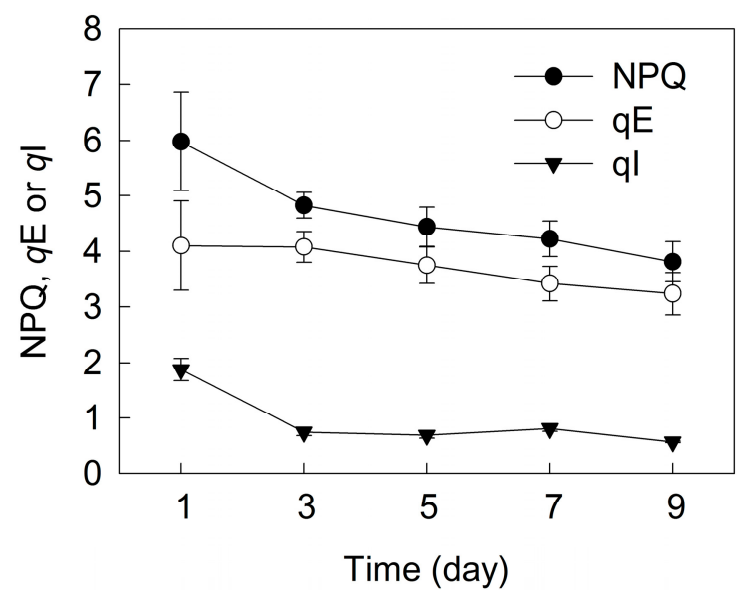

Figure 6. The NPQ (non-photochemical quenching), $q \mathrm{E}$ (fast component of NPQ) and $q \mathrm{I}$ (slow component of NPQ) during cell transformation and astaxanthin accumulation in H. pluvialis cultured in tubular photobioreactors. Mean \pm SE of five replicates are presented. 
Plastid terminal oxidase (PTOX) is the core protein of chlororespiration that oxidizes the plastoquinone $(\mathrm{PQ})$ pool. The level of PTOX did not change in the initial three days of incubation, and increased gradually over time after five days of incubation in H. pluvialis (Figure 7).

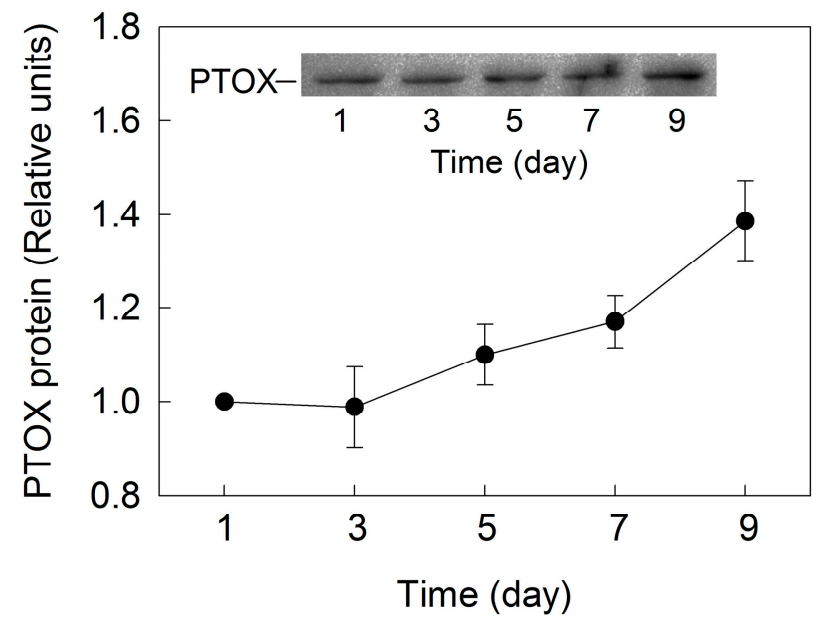

Figure 7. Densitometry of plastid terminal oxidase (PTOX) protein during cell transformation and astaxanthin accumulation in $H$. pluvialis cultured in tubular photobioreactors. Inset shows the levels of PTOX protein. Representative results of three independent replicates are shown.

A transient postillumination increase in chlorophyll fluorescence following light to dark transition is due to the reduction of PQ [23,24]. It shows that both the initial rate of the increasing phase and the amplitude of the postillumination increase in chlorophyll fluorescence in $H$. pluvialis did not change over time during the incubation (Figure 8).
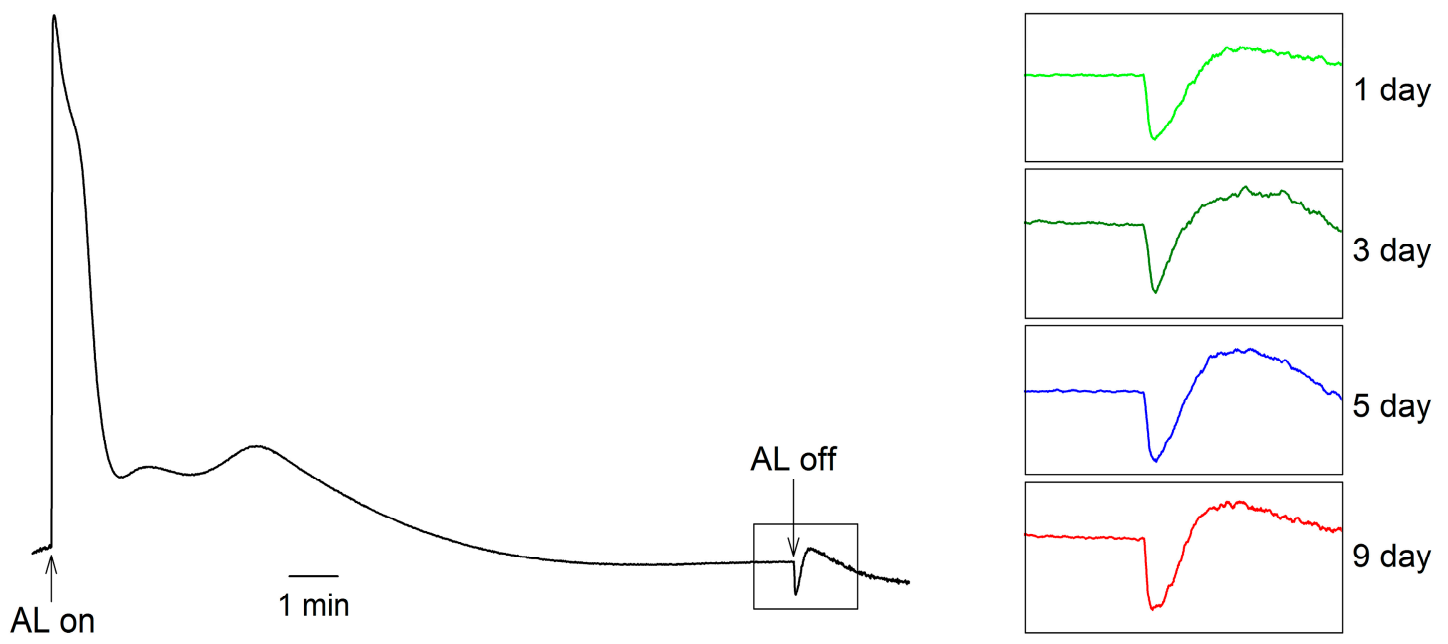

Figure 8. The postillumination increase in chlorophyll fluorescence during cell transformation and astaxanthin accumulation in $H$. pluvialis cultured in tubular photobioreactors. Insets show transient increase in chlorophyll fluorescence following light to dark transition. AL, white actinic light. Representative images from five independent replicates are shown in the figure.

As shown in Figure 9, the superoxide dismutase (SOD) activity did not change over time during the incubation. The activity of ascorbate peroxidase (APX) decreased sharply in the initial three days of incubation, and did not change over time after five days of incubation. The activities of catalase (CAT) 
increased in the initial three days of incubation, and decreased gradually over time after five days of incubation.

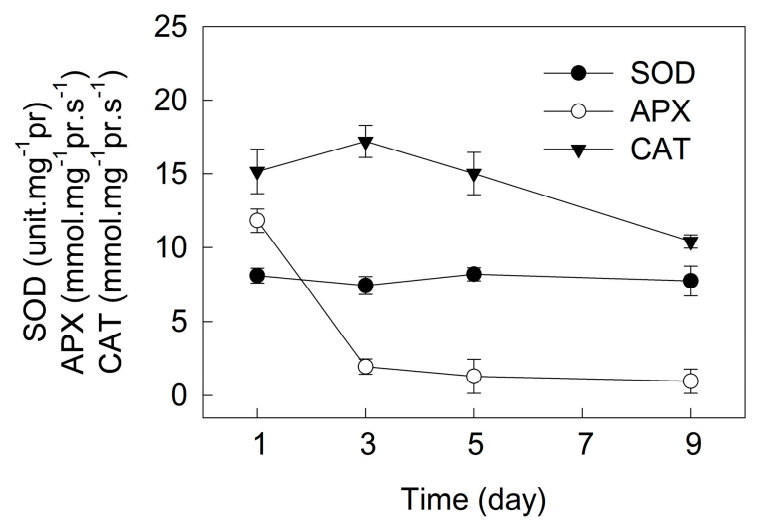

Figure 9. The activities of SOD (superoxide dismutase), APX (ascorbate peroxidase) and CAT (catalase) during cell transformation and astaxanthin accumulation in H. pluvialis cultured in tubular photobioreactors. Mean \pm SE of five replicates are presented.

Maximal photochemical efficiency of PSII $\left(F_{v} / F_{m}\right)$ is an indicator of photoinhibition in plants $[25,26]$. To examine the change in the capacity of photoprotection during the cell transformation and astaxanthin accumulation, we measured the change in $F_{v} / F_{m}$ in $H$. pluvialis at predawn (6:00) and at midday with photo flux density (PFD) approximately $2300 \mu \mathrm{mol} \cdot \mathrm{m}^{-2} \cdot \mathrm{s}^{-1}(12: 00)$ during the incubation (Figure 10). The $F_{v} / F_{m}$ value measured at predawn was maintained at relatively stable values in the initial five days of incubation and decreased only slightly after seven days of incubation. However, the $F_{v} / F_{m}$ measured at midday showed a considerable decrease before seven days of incubation, compared with that measured at predawn. The $F_{v} / F_{m}$ measured at midday decreased by $64.0 \%$ in $H$. pluvialis cells at one day, but did not change in cells at nine days, compared with that measured at predawn (Figure 10).

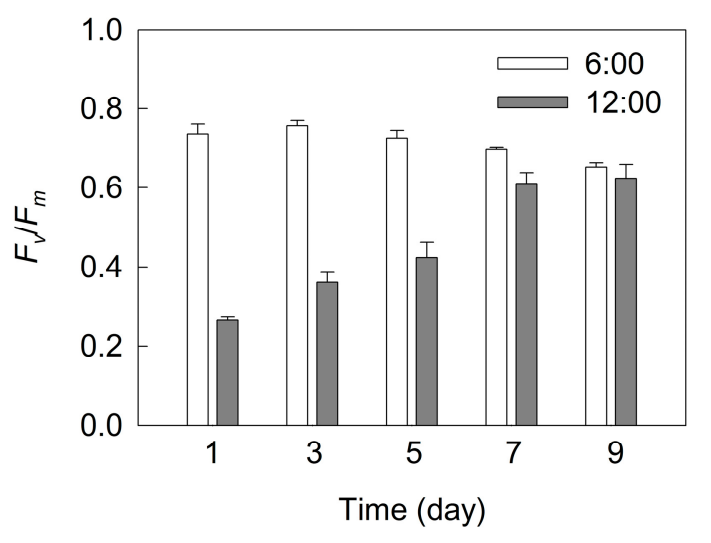

Figure 10. The maximal photochemical efficiency of photosystem II $\left(F_{v} / F_{m}\right)$ during cell transformation and astaxanthin accumulation in H. pluvialis grown in tubular photobioreactors. Samples were taken at predawn (6:00) and at midday with photo flux density (PFD) approximately $2300 \mu \mathrm{mol} \cdot \mathrm{m}^{-2} \cdot \mathrm{s}^{-1}$ (12:00). Mean $\pm \mathrm{SE}$ of five replicates are presented.

\section{Discussion}

The green motile cells transformed into non-motile cells during the first three days of incubation (cell transformation phase), with noticeable amounts of astaxanthin having accumulated by five days of incubation (astaxanthin accumulation phase), in H. pluvialis grown outdoors in tubular 
photobioreactors (Figure 1). Chlorophyll content increased gradually with time during the initial seven days of incubation, but decreased slightly after seven days of incubation, which indicates that the nine-day incubation period in this experiment was the early stage of astaxanthin accumulation in H. pluvialis grown outdoors. We speculate that chlorophyll content will decrease significantly after nine days of incubation (late stage of astaxanthin accumulation).

The photosynthetic capacity increased during cell transformation, and then decreased gradually during astaxanthin accumulation (Figure 2). Meanwhile, multiple changes of photosynthetic behaviors occurred during cell transformation and astaxanthin accumulation (Figure 3). In detail, during the initial three days of incubation, there was relative variable fluorescence kinetics at the J-step, the $L$-band and the electron transport per active reaction center (ETo/RC) did not change, the $K$-band, the light absorption per active reaction center (ABS/RC) and the trapping of excitation energy per active reaction center $(\mathrm{TRo} / \mathrm{RC})$ decreased, whereas the maximal amplitudes of fluorescence in the I-P phase of the OJIP transient, the efficiency of electron transport $\left(\psi_{0}\right)$ and the quantum yield of electron transport $\left(\varphi_{E o}\right)$ increased over time (Figures 3 and 4 ). These results indicate that the light absorption and photosynthetic electron transport became much more efficient $[17,22]$ during cell transformation, which was also indicated by the fact that the performance index $\mathrm{PI}_{\mathrm{ABS}}$ as well as its individual partial components RC/ABS, $\varphi_{P_{0}} /\left(1-\varphi_{P_{0}}\right)$ and $\psi_{o} /\left(1-\psi_{0}\right)$ increased significantly during the initial three days of incubation (Figure 4).

After five days of incubation, during astaxanthin accumulation (Figure 1), the fact that the maximal amplitude in the I-P phase of the OJIP transient decreased obviously over time (Figure 3E) indicates a decrease in the pool size of PSI end electron acceptors, leading to an over-reduction of the PSI acceptor side [17,22,27]. Moreover, the relative variable fluorescence kinetics at the J-step increased with increased time after five days of incubation (Figure 3B), which indicates that the ratio of $\mathrm{Q}_{\mathrm{A}}{ }^{-} / \mathrm{Q}_{\mathrm{A}}$ increased $[19,26]$, resulting in an over-reduction of the PSII acceptor side during astaxanthin accumulation.

The $L$-bands increased significantly over time after five days of incubation (Figure 3C), indicating that the PSII units were less grouped, resulting in a poorer utilization of the excitation energy and a lower stability of the system $[17,26]$ during astaxanthin accumulation in $H$. pluvialis. The K-bands (Figure 3D) increased significantly with increased time, which suggests that the donor side of PSII might be impaired $[15,17]$ during astaxanthin accumulation. Meanwhile, RC/ABS, $\varphi_{P_{0}} /\left(1-\varphi_{P_{0}}\right)$ and $\psi_{0} /\left(1-\psi_{0}\right)$ declined gradually during astaxanthin accumulation (Figure 4$)$. All these results indicate that the capacity and efficiency of photosynthetic energy utilization decreased significantly during astaxanthin accumulation in $\mathrm{H}$. pluvialis, leading to imbalance between photosynthetic light absorption and energy utilization. Moreover, the observation that ABS/RC increased but ETo/RC, $\psi_{o}$ and $\varphi_{E o}$ decreased with increased time after five days of incubation (Figure 4) also indicates that the balance between photosynthetic light absorption and energy utilization was disturbed $[17,22]$ during astaxanthin accumulation. The imbalance between light absorption and utilization will cause the over-excitation of PSII reaction centers [22,28].

The above results suggest that PSII activity increased during cell transformation phase but decreased gradually during the astaxanthin accumulation phase in H. pluvialis grown outdoors. The change of PsaA protein level (Figure 5) indicates that PSI activity also increased during the cell transformation phase but decreased gradually during astaxanthin accumulation phase in H. pluvialis. These results are consistent with a change of photosynthetic capacity (Figure 2).

Excess light energy will accelerate the generation of reactive oxygen species (ROS), leading to photoinhibition $[22,29]$. In order to escape from exposure to excess light, most plants have evolved various defence mechanisms to prevent the generation of ROS, such as NPQ [30,31], PTOX [32], PSI cyclic electron transport (CEF-PSI) [24,31] and antioxidant enzymes [33]. NPQ, of which the main component is $q \mathrm{E}$, can dissipate excess absorbed light energy in PSII. The $q \mathrm{E}$ mechanism is controlled by the xanthophyll cycle. During cell transformation and astaxanthin accumulation in H. pluvialis, the photoprotective capacity of NPQ decreased gradually (Figure 6). Interestingly, NPQ value in this 
experiment is much higher (close to 6 at 1 day) than that in other reports [13,14], likely due to the difference of growth light intensities of $H$. pluvialis.

PTOX can remove electrons from the PQ pool, which may have a significant role in dissipation of excess electrons under high light [32]. PTOX activation is also correlated to the carotenoid accumulation [13]. The level of PTOX did not change during cell transformation phase, and increased gradually during astaxanthin accumulation phase (Figure 7), which is consistent with the process of astaxanthin synthesis during the incubation in H. pluvialis grown outdoors. However, the role of PTOX (photoprotection and/or activation of astaxanthin synthesis) in H. pluvialis during cell transformation and astaxanthin accumulation needs to be further investigated.

A transient postillumination increase in chlorophyll fluorescence following light to dark transition is caused by the reduction of PQ. This reaction is correlated to the balance between oxidation of PQ by PTOX and reduction of PQ by CEF-PSI [23]. According to the change of PTOX activity (Figure 7), both the initial rate of the increasing phase and the amplitude of the postillumination increase in chlorophyll fluorescence did not change over time during the incubation in H. pluvialis (Figure 8), indicating that CEF-I activity did not change during the cell transformation phase and increased gradually during the astaxanthin accumulation phase. The CEF-I might play an important role in photoprotection of $H$. pluvialis cells during astaxanthin accumulation.

Under photoinhibitory conditions (high light or UV light), there are two major sites of ROS generation in chloroplasts: the end of photosynthetic electron transport chain (acceptor side of PSI) and the PSII reaction centers $[22,28,31,34]$. The over-reduction of the PSI acceptor side and the over-excitation of PSII reaction centers during astaxanthin accumulation in H. pluvialis would inevitably enhance the generation of ROS, leading to more severe photoinhibition under high light. Meanwhile, the decrease in photosynthetic capacity during astaxanthin accumulation (Figure 2) also accelerated the generation of excess light energy. However, we compared the $F_{v} / F_{m}$ value at predawn and at midday during the incubation of $H$. pluvialis (Figure 10). The results demonstrate that photoinhibition was more severe during cell transformation because of the decrease in photoprotective capacity, and the level of photoinhibition decreased significantly over time during astaxanthin accumulation, especially after seven days of incubation. This is reflected by the fact that $F_{v} / F_{m}$ determined at midday decreased significantly during the initial three days of incubation, but was affected very little after seven days of incubation, compared with that determined at predawn (Figure 10). It has been suggested that astaxanthin can protect $H$. pluvialis cells against oxidative stress $[35,36]$. In our previous study, the role of two distinct antioxidative mechanisms, the defensive enzyme system and the astaxanthin were compared in H. pluvialis [37]. As an antioxidant, astaxanthin is more efficient than the defensive enzyme system. In the present study, SOD activity did not change over time during cell transformation and astaxanthin accumulation. The activity of APX decreased sharply during cell transformation, and did not change during astaxanthin accumulation. The activity of CAT increased during cell transformation and decreased gradually during astaxanthin accumulation (Figure 9). Therefore, in H. pluvialis grown outdoors, NPQ, PTOX, CEF-I, defensive enzymes (SOD, APX and CAT) activities and the accumulation of amounts of astaxanthin can protect cells against photoinhibition.

Moreover, it appears that in the initial three days of incubation (cell transformation phase), the high light at midday could lead to more severe photoinhibition and even decrease the cell numbers in cultures of green motile $H$. pluvialis. Therefore, in large-scale cultivation, the effective shade can protect green motile $H$. pluvialis cells against photoinhibition, thereby enhancing biomass for astaxanthin production.

\section{Materials and Methods}

\subsection{Strains and Culture Conditions}

The alga Haematococcus pluvialis (strain $H_{0}$ ) was obtained from the Institute of Oceanology, Chinese Academy of Sciences, Qingdao, China. The seeding culture (the motile green vegetative cells) 
was prepared by using an exponentially growing culture of $H$. pluvialis grown outdoors in modified MCM medium (200 mg. $\left.\mathrm{L}^{-1} \mathrm{NO}_{3}{ }^{-}\right)[37,38]$ in closed column photobioreactors, which was re-cultured in nitrogen-limited medium (50 mg. $\left.\mathrm{L}^{-1} \mathrm{NO}_{3}{ }^{-}\right)$up to a final concentration of $1.7 \times 10^{5}$ cells $\cdot \mathrm{mL}^{-1}$ in tubular photobioreactors (working volume $5000 \mathrm{~L}$ ) outdoors in the afternoon (17:00). H. pluvialis cells incubated for 1, 3, 5, 7 and 9 days were used for all analyses. All of these experiments were done in the Yunnan Alphy Biotech Co., Ltd. (Chuxiong, China) facilities. During this period, there was typical Yunnan winter weather, with a mean daily air temperature between 17 and $21^{\circ} \mathrm{C}$ and an average $\mathrm{PFD}$ of around $2300 \mu \mathrm{mol} \cdot \mathrm{m}^{-2} \cdot \mathrm{s}^{-1}$ at midday. The pigments, respiration rate, $\mathrm{O}_{2}$ evolution rate, PsaA protein level and chlorophyll $a$ fluorescence (OJIP) transients were determined at predawn (6:00). The NPQ, PTOX protein level, postillumination increase in chlorophyll fluorescence and antioxidant enzyme (superoxide dismutase, ascorbate peroxidase and catalase) activities were determined at midday (12:00). The maximal photochemical efficiency of PSII $\left(F_{v} / F_{m}\right)$ was determined at predawn and at midday (12:00).

\subsection{Analytical Procedures}

Algal astaxanthin was extracted with methanol/dichloromethane $(1: 3, v / v)$, and the extracts were analyzed by HPLC (Agilent, Santa Clara, CA, USA) following the protocol reported by Yuan et al. [39]. Algal chlorophyll was extracted with $80 \%$ acetone, and the extracts were analyzed with a UV-120 system (Shimadzu, Kyoto, Japan) according to the method of Porra [40].

The total photosynthetic $\mathrm{O}_{2}$ evolution rate and respiration rate were measured with a Clark-type $\mathrm{O}_{2}$ electrode (DW2, Hansatech, Norfolk, UK) according to Zhang and Liu [18].

Chlorophyll $a$ fluorescence (OJIP) transients of cells during the incubation were measured with a Handy PEA fluorometer (Hansatech). All measurements were performed with $10 \mathrm{~min}$ dark-adapted cells. Fluorescence levels $F_{o}(50 \mu \mathrm{s}), F_{K}(300 \mu \mathrm{s}), F_{J}(2 \mathrm{~ms}), F_{I}(30 \mathrm{~ms})$, and $F_{P}$ (peak, about $300 \mathrm{~ms}$ ) were recorded. The relative variable fluorescence kinetics $\left(W_{O P}, W_{O K}, W_{O J}\right.$, and $\left.W_{O I}\right)$ were obtained by normalizing the fluorescence transients according to the equations of the JIP-test $[17,20,21]$. Difference kinetics between the cells incubated for $1 \mathrm{~d}$ and other samples were calculated as: $\Delta W=W-W_{\text {(cells incubated for } 1 \text { day) }}$, where $W$ was the relative variable fluorescence. In addition, the following parameters were used for the quantification of PSII behaviors [17,41]:

(1) The specific energy fluxes, i.e. the light absorption per active reaction center ( $A B S / R C$, the average antenna size), the trapping of excitation energy per active reaction center (TRo/RC), the electron transport per active reaction center (ETo/RC).

(2) The flux ratios or yields, i.e., the efficiency of electron transport $\left(\psi_{0}\right)$, and the quantum yield of electron transport $\left(\varphi_{E o}\right)$.

(3) The photosynthetic performance index $\mathrm{PI}_{\mathrm{ABS}}$ as well as its individual partial components, the efficiency of light absorption (RC/ABS), the performance due to the quantum efficiency of primary photochemistry $\left(\varphi_{P_{0}} /\left(1-\varphi_{P_{0}}\right)\right)$ and the performance due to the quantum efficiency of the conversion of excitation energy to electron transport $\left(\psi_{0} /\left(1-\psi_{0}\right)\right)$, were also calculated according to the JIP-test equations.

The NPQ were measured with a FMS-2 pulse-modulated fluorometer (Hansatech, Norfolk, UK) as described in Jiang et al. [42]. Components of NPQ, including a fast component $(q \mathrm{E})$ and a slow component $(q \mathrm{I})$, were determined following the protocol of Johnson et al. [43].

The PsaA and PTOX protein was detected by Western Blot according to Fan et al. [44]. The total proteins were denatured and separated using a $12 \%$ polyacrylamide gradient gel. The denatured protein complexes in the gel were then electro-blotted to polyvinylidene fluoride (PVDF) membranes, probed with PsaA or PTOX antibody, and then visualized by the enhanced chemiluminescence method. The quantitative image analysis of protein levels was performed with Gel-Pro Analyzer 4.0 software (Media Cybernetics, Bethesda, MD, USA). 
The postillumination increase in chlorophyll fluorescence was measured with an FMS-2 pulse-modulated fluorometer (Hansatech, Norfolk, UK) according to Wang et al. [24].

The activities of superoxide dismutase (SOD; EC 1.15.1.1), ascorbate peroxidase (APX; EC 1.11.1.11) and catalase (CAT; EC 1.11.1.6) were determined according to Jia et al. [45].

Maximal photochemical efficiency of PSII $\left(F_{v} / F_{m}\right)$ of cultures during incubation was measured using a Handy PEA fluorometer (Hansatech, Norfolk, UK) [26]. $F_{v} / F_{m}$ measurement was performed using cultures that had been dark-adapted for $10 \mathrm{~min}$ prior to doing the measurement.

Least significant difference (LSD) was used to analyze differences between the measurements.

\section{Conclusions}

In H. pluvialis grown outdoors in tubular photobioreactors, during the cell transformation phase, the light absorption and photosynthetic electron transport became much more efficient, and the capacities of photoprotective mechanisms decreased. During astaxanthin accumulation, the pool size of PSI end electron acceptors decreased significantly, leading to an over-reduction of the PSI acceptor side. The balance between photosynthetic light absorption and energy utilization was disturbed, resulting in the over-excitation of PSII reaction centers. The over-reduction of the PSI acceptor side and the over-excitation of the PSII reaction centers during astaxanthin accumulation would inevitably enhance the generation of ROS, leading to more severe photoinhibition under high light, e.g., at midday. However, the capacity for photoprotection in H. pluvialis increased significantly with time during astaxanthin accumulation, which might be because the NPQ, PTOX, CEF-I, defensive enzyme (SOD, APX and CAT) activities and the accumulated astaxanthin can protect cells against photoinhibition.

Acknowledgments: This study was supported by the China National Nature Science Foundation $(31572639,31300330)$ and Laboratory for Marine Biology and Biotechnology, Qingdao National Laboratory for Marine Science and Technology, Qingdao China. Special thanks are given to John Van Der Meer (Pan-American Marine Biotechnology Association, Halifax, Canada) for his assistance with proofreading.

Author Contributions: Litao Zhang and Jianguo Liu designed the study and wrote the manuscript; Litao Zhang, Fang Su and Chunhui Zhang performed the experiments and analyzed the data; Fengying Gong determined the levels of PTOX and PsaA protein.

Conflicts of Interest: The authors declare no conflict of interest.

\section{References}

1. Chen, G.Q.; Wang, B.B.; Han, D.X.; Sommerfeld, M.; Lu, Y.H.; Chen, F.; Hu, Q. Molecular mechanisms of the coordination between astaxanthin and fatty acid biosynthesis in Haematococcus pluvialis (Chlorophyceae). Plant J. 2015, 81, 95-107. [CrossRef] [PubMed]

2. Giannelli, L.; Yamada, H.; Katsuda, T.; Yamaji, H. Effects of temperature on the astaxanthin productivity and light harvesting characteristics of the green alga Haematococcus pluvialis. J. Biosci. Bioeng. 2015, 119, 345-350. [CrossRef] [PubMed]

3. Wang, J.F.; Han, D.X.; Sommerfeld, M.R.; Lu, C.M.; Hu, Q. Effect of initial biomass density on growth and astaxanthin production of Haematococcus pluvialis in an outdoor photobioreactor. J. Appl. Phycol. 2013, 25, 253-260. [CrossRef]

4. Benemann, J.R. Microalgae aquaculture feeds. J. Appl. Phycol. 1992, 4, 233-245. [CrossRef]

5. Sommer, T.R.; Potts, W.T.; Morrissy, N.M. Utilization of microalgal astaxanthin by rainbow trout (Oncorhyncus mykiss). Aquaculture 1991, 94, 79-88. [CrossRef]

6. Goswami, G.; Chaudhuri, S.; Dutta, D. The present perspective of astaxanthin with reference to biosynthesis and pharmacological importance. World J. Microbiol. Biotechnol. 2010, 26, 1925-1939. [CrossRef]

7. Guerin, M.; Huntley, M.E.; Olaizola, M. Haematococcus astaxanthin: Applications for human health and nutrition. Trends Biotechnol. 2003, 21, 210-216. [CrossRef]

8. Liu, J.G.; van der Meer, J.P.; Zhang, L.T.; Zhang, Y. Cultivation of Haematococcus pluvialis for astaxanthin production. In Micro-Algal Production for Biomass and High-Value Products; Slocombe, S.P., Benemann, J.R., Eds.; Taylor \& Francis: New York, NY, USA, 2016; pp. 267-293. 
9. Kobayashi, M.; Kakizono, T.; Nishio, N.; Nagai, S. Effects of light intensity, light quality, and illumination cycle on astaxanthin formation in a green alga, Haematococcus pluvialis. J. Ferment. Bioeng. 1992, 74, 61-63. [CrossRef]

10. Steinbrenner, J.; Linden, H. Regulation of two carotenoid biosynthesis genes coding for phytoene synthase and carotenoid hydroxylase during stress-induced astaxanthin biosynthesis in the green alga Haematococcus pluvialis. Plant Physiol. 2000, 125, 810-817. [CrossRef]

11. Steinbrenner, J.; Linden, H. Light induction of carotenoid biosynthesis genes in the green alga Haematococcus pluvialis: Regulation by photosynthetic redox control. Plant Mol. Biol. 2003, 52, 343-356. [CrossRef] [PubMed]

12. Boussiba, S. Carotenogenesis in the green alga Haematococcus pluvialis: Cellular physiology and stress response. Physiol. Plant. 2000, 108, 111-117. [CrossRef]

13. Scibilia, L.; Girolomoni, L.; Berteotti, S.; Alboresi, A.; Ballottari, M. Photosynthetic response to nitrogen starvation and high light in Haematococcus pluvialis. Algal Res. 2015, 12, 170-181. [CrossRef]

14. Chekanov, K.; Lukyanov, A.; Boussiba, S.; Aflalo, C.; Solovchenko, A. Modulation of photosynthetic activity and photoprotection in Haematococcus pluvialis cells during their conversion into haematocysts and back. Photosynth. Res. 2016, 128, 313-323. [CrossRef] [PubMed]

15. Chen, S.G.; Strasser, R.J.; Qiang, S. In vivo assessment of effect of phytotoxin tenuazonic acid on PSII reaction centers. Plant Physiol. Biochem. 2014, 84, 10-21. [CrossRef] [PubMed]

16. Kalaji, H.M.; Oukarroum, A.; Alexandrov, V.; Kouzmanova, M.; Brestic, M.; Zivcak, M.; Samborska, I.A.; Cetner, M.D.; Allakhverdiev, S.I.; Goltsev, V. Identification of nutrient deficiency in maize and tomato plants by in vivo chlorophyll $a$ fluorescence measurements. Plant Physiol. Biochem. 2014, 81, 16-25. [CrossRef] [PubMed]

17. Yusuf, M.A.; Kumar, D.; Rajwanshi, R.; Strasser, R.J.; Tsimilli-Michael, M.; Sarin, N.B. Overexpression of $\gamma$-tocopherol methyl transferase gene in transgenic Brassica juncea plants alleviates abiotic stress: Physiological and chlorophyll $a$ fluorescence measurements. Biochim. Biophys. Acta Bioenerg. 2010, 1797, 1428-1438. [CrossRef] [PubMed]

18. Zhang, L.T.; Liu, J.G. Effects of heat stress on photosynthetic electron transport in a marine cyanobacterium Arthrospira sp. J. Appl. Phycol. 2016, 28, 757-763. [CrossRef]

19. Strasser, B.J.; Strasser, R.J. Measuring fast fluorescence transients to address environmental questions: The JIP-test. In Photosynthesis: From Light to Biosphere; Mathis, P., Ed.; Kluwer Academic Publishers Press: Dordrecht, The Netherlands, 1995; pp. 977-980.

20. Strasser, R.J.; Srivatava, A.; Tsimilli-Michael, M. The fluorescence transient as a tool to characterize and screen photosynthetic samples. In Probing Photosynthesis: Mechanism, Regulation and Adaptation; Yunus, M., Pathre, U., Mohanty, P., Eds.; Taylor \& Francis: London, UK, 2000; pp. 445-483.

21. Strasser, R.J.; Tsimilli-Michael, M.; Srivastava, A. Analysis of the chlorophyll $a$ fluorescence transient. In Chlorophyll Fluorescence: A Signature of Photosynthesis; Papageorgiou, G.C., Ed.; Kluwer Academic Publishers Press: Dordrecht, The Netherlands, 2004; pp. 321-362.

22. Zhang, L.T.; Zhang, Z.S.; Gao, H.Y.; Xue, Z.C.; Yang, C.; Meng, X.L.; Meng, Q.W. Mitochondrial alternative oxdiase pathway protects plants against photoinhibition by alleviating inhibition of the repair of photodamaged PSII through preventing formation of reactive oxygen species in Rumex K-1 leaves. Physiol. Plant. 2011, 143, 396-407. [CrossRef] [PubMed]

23. Muraoka, R.; Okuda, K.; Kobayashi, Y.; Shikanai, T. A eukaryotic factor required for accumulation of the chloroplast NAD(P)H dehydrogenase complex in arabidopsis. Plant Physiol. 2006, 142, 1683-1689. [CrossRef] [PubMed]

24. Wang, P.; Duan, W.; Takabayashi, A.; Endo, T.; Shikanai, T.; Ye, J.Y.; Mi, H.L. Chloroplastic NAD(P)H dehydrogenase in tobacco leaves functions in alleviation of oxidative damage caused by temperature stress. Plant Physiol. 2006, 141, 465-474. [CrossRef] [PubMed]

25. Takahashi, S.; Milward, S.E.; Fan, D.Y.; Chow, W.S.; Badger, M.R. How does cyclic electron flow alleviate photoinhibition in Arabidopsis? Plant Physiol. 2009, 149, 1560-1567. [CrossRef] [PubMed]

26. Zhang, L.T.; He, M.L.; Liu, J.G.; Li, L. Role of the mitochondrial alternative oxidase pathway in hydrogen photoproduction in Chlorella protothecoides. Planta 2015, 241, 1005-1014. [CrossRef] [PubMed] 
27. Kalachanis, D.; Manetas, Y. Analysis of fast chlorophyll fluorescence rise (O-K-J-I-P) curves in green fruits indicates electron flow limitations at the donor side of PSII and the acceptor sides of both photosystems. Physiol. Plant. 2010, 139, 313-323. [CrossRef] [PubMed]

28. Vass, I. Role of charge recombination processes in photodamage and photoprotection of the photosystem II complex. Physiol. Plant. 2011, 142, 6-16. [CrossRef] [PubMed]

29. Horton, P.; Ruban, A.V.; Walters, R.G. Regulation of light harvesting in green plants. Annu. Rev. Plant Physiol. Mol. Biol. 1996, 47, 655-684. [CrossRef] [PubMed]

30. Müller, P.; Li, X.P.; Niyogi, K.K. Non-photochemical quenching. A response to excess light energy. Plant Physiol. 2001, 125, 1558-1566. [CrossRef] [PubMed]

31. Niyogi, K.K. Safety valves for photosynthesis. Curr. Opin. Plant Biol. 2000, 3, 455-460. [CrossRef]

32. Rumeau, D.; Peltier, G.; Cournac, L. Chlororespiration and cyclic electron flow around PSI during photosynthesis and plant stress response. Plant Cell Environ. 2007, 30, 1041-1051. [CrossRef] [PubMed]

33. Mittler, R. Oxidative stress, antioxidants and stress tolerance. Trends Plant Sci. 2002, 7, 405-410. [CrossRef]

34. Niyogi, K.K. Photoprotection revisited: Genetic and molecular approaches. Annu. Rev. Plant Physiol. Plant Mol. Biol. 1999, 50, 333-359. [CrossRef] [PubMed]

35. Kobayashi, M. In vivo antioxidant role of astaxanthin under oxidative stress in the green alga Haematococcus pluvialis. Appl. Microbiol. Biotechnol. 2000, 54, 550-555. [CrossRef] [PubMed]

36. Kobayashi, M.; Okada, T. Protective role of astaxanthin against u.v.-B irradiation in the green alga Haematococcus pluvialis. Biotechnol. Lett. 2000, 22, 177-181. [CrossRef]

37. Liu, J.G.; Zhang, X.L.; Sun, Y.H.; Lin, W. Antioxidative capacity and enzyme activity in Haematococcus pluvialis cells exposed to superoxide free radicals. Chin. J. Oceanol. Limnol. 2010, 28, 1-9. [CrossRef]

38. Zhang, C.H.; Zhang, L.T.; Liu, J.G. The role of photorespiration during astaxanthin accumulation in Haematococcus pluvialis (Chlorophyceae). Plant Physiol. Biochem. 2016, 107, 75-81. [CrossRef] [PubMed]

39. Yuan, J.; Chen, F.; Liu, X.; Li, X. Carotenoid composition in the green microalga Chlorococcum. Food Chem. 2002, 76, 319-325. [CrossRef]

40. Porra, R.J. The chequered history of the development and use of simultaneous equations for the accurate determination of chlorophylls $a$ and $b$. Photosynth. Res. 2002, 73, 149-156. [CrossRef] [PubMed]

41. Wen, X.G.; Qiu, N.W.; Lu, Q.T.; Lu, C.M. Enhanced thermotolerance of photosystem II in salt-adapted plants of the halophyte Artemisia anethifolia. Planta 2005, 220, 486-497. [CrossRef] [PubMed]

42. Jiang, C.D.; Gao, H.Y.; Zou, Q.; Jiang, G.M.; Li, L.H. Leaf orientation, photorespiration and xanthophyll cycle protect young soybean leaves against high irradiance in field. Environ. Exp. Bot. 2004, 55, 87-96. [CrossRef]

43. Johnson, G.N.; Young, A.J.; Scholes, J.D.; Horton, P. The dissipation of excess excitation energy in British plant species. Plant Cell Environ. 1993, 16, 673-679. [CrossRef]

44. Fan, X.L.; Zhang, Z.S.; Gao, H.Y.; Yang, C.; Liu, M.J.; Li, Y.T.; Li, P.M. Photoinhibition-like damage to the photosynthetic apparatus in plant leaves induced by submergence treatment in the dark. PLoS ONE 2014, 9, e89067. [CrossRef] [PubMed]

45. Jia, Y.J.; Cheng, D.D.; Wang, W.B.; Gao, H.Y.; Liu, A.X.; Li, X.M.; Meng, Q.W. Different enhancement of senescence induced by metabolic products of Alternaria alternata in tobacco leaves of different ages. Physiol. Plant. 2010, 138, 164-175. [CrossRef] [PubMed]

(C) 2016 by the authors; licensee MDPI, Basel, Switzerland. This article is an open access article distributed under the terms and conditions of the Creative Commons Attribution (CC-BY) license (http://creativecommons.org/licenses/by/4.0/). 